\title{
The interface between clinicians and laboratory staff: A field study in northern Tanzania
}

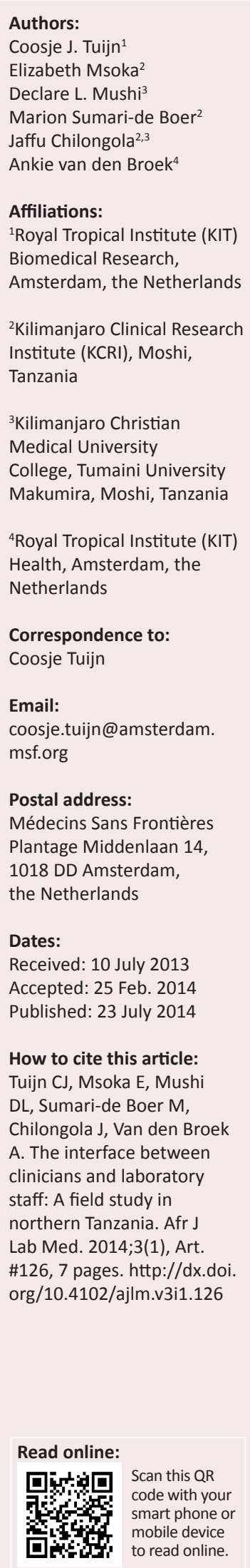

Background: Strengthening the communication and professional relationships between clinicians and laboratory workers is essential in order to positively change clinicians' attitudes about the reliability of diagnostic tests, enhancing the use of laboratory diagnostics and, ultimately, improving patient care. We developed an analytical framework to gain insight into the factors that influence communication amongst health professionals.

Objective: To explore whether the interaction between clinicians and laboratory workers influences the use of laboratory test results in clinical decision making.

Methods: Four health facilities in northern Tanzania were selected using convenience sampling, whereas study participants were selected using purposive sampling. The quantitative and qualitative data collection methods included self-administered questionnaires; semistructured, individual interviews; in-depth, individual interviews; and/or focus group discussions with clinicians and laboratory workers. Thematic content analyses were performed on qualitative data based on the framework. Descriptive statistical analyses of quantitative data were conducted using Microsoft Excel.

Results: Contact between clinicians and laboratory professionals is seldom institutionalised and collaboration is rare. The clinicians believe collaboration with laboratory staff is a challenge because of the gap in education levels. Laboratory workers' education levels are often lower than their positions require, leading to clinicians' lack of respect for and confidence in laboratory professionals, which compromises the laboratory staff's motivation.

Conclusions: Hospital managers, clinicians and laboratory workers need to recognise the critical and complementary roles each professional plays and the importance of addressing the gap between them. Field application of the framework proved successful, justifying the expansion of this study to a larger geographical area to include additional healthcare institutions.

\section{Introduction}

Medical laboratories play a significant role in the diagnosis, monitoring and treatment of diseases; yet the efficacy of the information they provide may be questioned because of several factors, including the capacity of the laboratory workforce, the laboratory infrastructure and the availability of equipment and materials, especially in low-income countries. Whilst improving the quality of laboratories is a solution, it does not always result in proper execution of tests. . $^{1,2,3}$ Other obstacles that must also be considered are the cultural beliefs of the patients, attrition of healthcare workers, physicians' attitudes and inadequate supplies of consumables. ${ }^{4}$

Medical laboratory services offer essential information for diagnoses and/or treatment plans. The communication and interactions between laboratory and clinical health workers can influence physicians' request behaviour and treatment interventions. Previous studies have shown that lack of communication is a barrier to effective healthcare ${ }^{5,6,7,8,9}$ Improved communication between clinicians and laboratory workers is essential to changing clinicians' attitudes about the reliability of diagnostic tests, possibly leading to increased use of laboratory diagnostics and, ultimately, improving patient care. ${ }^{5}$

This interface between clinicians and laboratory health workers is complex; the two groups may communicate face-to-face or by request and result forms, phone calls, text messages, e-mails or computerised forms. The factors that influence the mode of communication and shape the relationship between these two professional groups require further exploration. For this reason we constructed an analytical framework based on existing literature. $10,11,12,13,14,15$ After further literature searches, analysis of guidelines for laboratories and discussion with experts, a conceptual model was developed. ${ }^{16}$ The model addresses the phases where clinicians and laboratory workers

Copyright:@ 2014. The Authors. Licensee: AOSIS OpenJournals. This work is licensed under the Creative Commons Attribution License. 
interact; the organisational and personal factors affecting their interface; and the socio-political, economic and cultural environment within which the health facility operates. The objective of this study was to demonstrate and test the analytical framework and to gain insight into the relationship between clinicians and laboratory workers and into the factors that influence their interface, with the intention of later scaling up the study using a calculated sample size. The analytical framework includes three phases of communication (pre-analytical, analytical, post-analytical) during which clinicians and laboratory workers interact (Tables $1 \mathrm{a}$ and $1 \mathrm{~b}) .{ }^{15}$ The testing process starts with a clinician ordering a test and sample collection, known as the pre-analytical phase. During the analytical phase, the sample is processed and analysed by laboratory staff. The post-analytical phase includes transfer of results from the laboratory back to the clinician. Each phase consists of organisational factors, subdivided into 'identity' and 'management', as well as personal factors, subdivided into 'individual' and 'professional'. The primary aim of the study was to explore whether the interaction between clinicians and laboratory workers influences the use of laboratory test results in clinical decision making. By means of the framework quantitative and qualitative tools were designed. The results of this study provide information on the importance of the interface between clinicians and laboratory workers and may form a basis for larger studies in the future. The implications of our findings are useful for health institutions in any country.

\section{Research method and design Study design}

This was an exploratory study, employing both quantitative and qualitative methods. Its purpose was to use tools to test the analytical framework and to better understand the factors that influence the interaction between clinical and laboratory workers. Most participants took part in a focus group discussion (FGD) immediately following completion of an anonymous, self-administered questionnaire (SAQ). If there were fewer than three participants for a FGD, in-depth, individual interviews were conducted. Semi-structured, individual interviews were used for hospital directors and heads of departments. FGDs and in-depth, individual interviews followed the same format and covered the same topics. The assessment of the data collection tools was done at a private, not-for-profit, faith-based, district hospital, where a group of clinicians and laboratory staff were invited to assess the tools.

\section{Study population}

The study population included hospital directors, heads of clinical and laboratory departments, clinicians and laboratory staff. Staff came from three categories of health facilities: private, government; faith-based not-for-profit; and private for-profit. Four hospitals participated in the study: a non-government referral hospital with 450 beds; a

TABLE 1a: Analytical framework. This framework was developed to test our conceptual model, ${ }^{16}$ and displays the organisational and personal factors playing a role during the three phases where clinicians and laboratory workers interact: pre-analytical, analytical and post-analytical.

\begin{tabular}{ll}
\hline Identity and management (Organisational factors) & Individual and professional factors (Personal factors) \\
\hline Management style: & The characteristics of the clinical and laboratory workforce of the organisation: \\
- Planning and implementation of regular meetings & - Age \\
- Monitoring and supervision systems & - Level of education \\
- Employment policies & - CPD opportunities \\
- Working environment & - Years in service \\
\hline- - & - Position in the organisation \\
\hline
\end{tabular}

CPD, Continuing Professional Development.

TABLE 1b: Analytical framework. This framework was developed to test our conceptual model, ${ }^{16}$ and displays the three phases where clinicians and laboratory workers interact: pre-analytical, analytical and post-analytical. Each phase consists of organisational and personal factors (Table 1a).

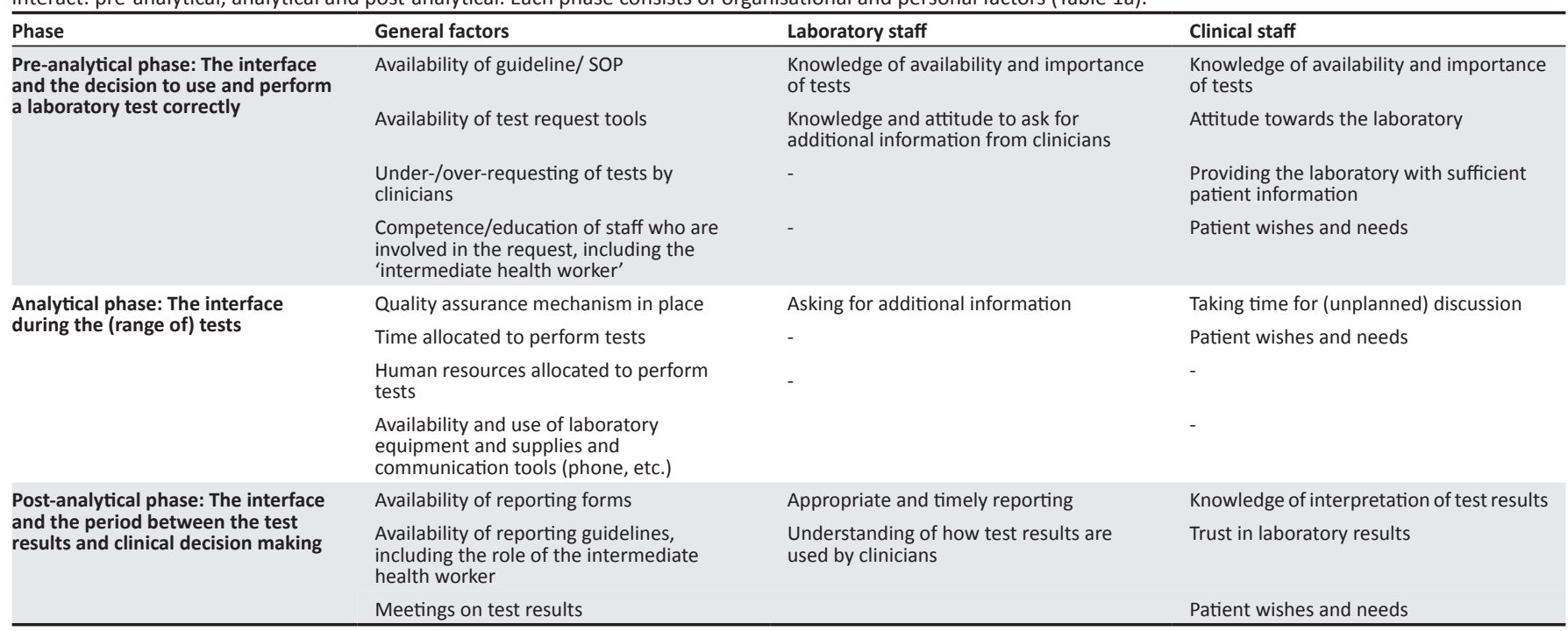


private not-for-profit hospital with 150 beds; a government regional hospital with 300 beds; and a private for-profit health centre with 50 beds.

\section{Sample size, sampling procedures and data collection}

Study sites were selected using convenience sampling, whereas study participants were selected using purposive sampling. As this was a pilot study, we did not determine a sample size. We contacted the sites and received verbal consent from the hospital directors for their participation. The interviewed staff members all signed written informed consent forms. Initially, a total of 48 staff members were asked to participate, including six clinicians and six laboratory workers from each of the four sites. However, at the time the study was conducted, only 35 staff members were present: 18 clinicians and 17 laboratory personnel. Amongst the latter were laboratory assistants and attendants who often perform the routine tests, namely, those who interacted most with clinicians (Tables 2a and 2b). SAQs were used to collect data from clinicians and laboratory staff at each hospital. After filling in the SAQs, staff members participated in either an FGD or an in-depth, individual interview, allowing for the opportunity to elaborate on the SAQs and further share their views. Interviews were carried out with hospital directors and heads of departments. All interviews and FGDs supplemented the SAQs and were conducted in Kiswahili for laboratory staff and in English for clinicians. Interviews were recorded via tape recorder or note taking. As outlined in the study protocol, FGDs involved six to 12 clinicians and three to nine laboratory staff members. These numbers were predetermined and agreed upon by the study team.

\section{Data analysis}

Data were collected within a period of five working days at the end of November 2011 and analysis was carried out throughout 2012. The individual interviews and FGDs were transcribed, translated and analysed by a social scientist and a research nurse. Qualitative data were analysed independently and manually, using a thematic framework approach involving data familiarisation, coding and development and categorisation of themes. Coding of collected qualitative data was driven by the developed framework (Tables $1 \mathrm{a}$ and $1 \mathrm{~b}$ ), whereby common words were sorted together, following an inductive method of code-creation. Once a theme was identified and reviewed, categorisation and corresponding codes were developed to sort and organise the data. After reading through the data, the two independent researchers discussed the codes and themes until they agreed on each one. Quotations were used to support and clarify the information provided using an editing analysis style. Descriptive statistical analysis of quantitative data from the structured questionnaires were carried out using Microsoft Excel.

\section{Ethical considerations}

Ethical clearance was obtained from the Kilimanjaro Christian Medical University College Research and the Ethical Review Committee of Tumaini University, Makumira (Research
Ethical Clearance certificate number 448 from Research Proposal 467). Verbal and written consent for the SAQs, interviews and FGDs was provided by study participants. Confidentiality was assured at all stages of this study, through the use of coding for sites and participants. No names or data can be traced back to individual participants.

\section{Trustworthiness}

The results of this study are based on actual findings as described in the research method and design section. Qualitative research (FGDs and in-depth interviews) were complementary to results obtained from the SAQ. The experimental design of this exploratory study is reliable and valid and the procedures of qualitative and quantitative research used in this study are according to standardised methods, as laid out by Varkevisser, Pathmanathan and Brownlee. ${ }^{17}$

\section{Results Participants}

Pre-testing of the data collection tools, firstly, through discussions with clinical and laboratory staff at a referral hospital and secondly, by group discussions at a district hospital, allowed researchers to strengthen and modify the data collection methods. In total, 35 questionnaires were administered to 18 clinicians and 17 laboratory staff members, an estimated one-third of the official staff number, according to the heads of departments.

\section{Factors influencing the interface Organisational factors}

Management factors: The analysis of the SAQs showed that 11 of the 18 clinicians $(61.1 \%)$ and eight of the 17 laboratory staff (47.1\%) were aware of the availability of rules and guidelines for requesting tests and reporting results. Of those remaining, one clinician (5.5\%) and nine laboratory staff (52.9\%) said there were no guidelines, whilst six clinicians (33.3\%) did not know whether or not guidelines existed. These findings were also evident in the FGDs. Those who were aware that there are guidelines in place noted that there is little time to adhere to them because of staffing shortages and an insufficient supply of reagents. Other factors that the study group cited as impacting on communication in relation to management of the organisation included the clinicians' doubts about laboratory test results and uncertainty as to whether Standard Operational Procedures are followed as well as the awareness of the persons to whom clinicians and laboratory staff report (Figure 1 and Figure 2). Furthermore, the lack of competent and highly-educated laboratory staff was cited by clinicians as being a barrier to effective communication; in many health facilities, only laboratory attendants and assistants are present to perform tests and they sometimes lack the communication skills of a more highly-educated laboratory technician. In FGDs with laboratory workers, it was noted that clinicians do not always use the test results with which they are provided. Patients sometimes ask clinicians to prescribe 
TABLE 2a: The demographic distribution related to the personal identities of the clinicians participating in this field study.

\begin{tabular}{lcc}
\hline Demographic factors & \multicolumn{2}{c}{ Gender } \\
\cline { 2 - 3 } & Male $(\boldsymbol{N}=\mathbf{1 1})$ & Female $(\boldsymbol{N}=\mathbf{7})$ \\
\hline Ethnicity & 7 & 3 \\
Chagga & 1 & 1 \\
Pare & 1 & 0 \\
Masai & 0 & 1 \\
Makonde & 1 & 1 \\
Kalenjin & 1 & 0 \\
Ugandan/Maganda & 0 & 1
\end{tabular}

Religion

Catholic

Lutheran

Muslim

Pentacostal

Seventh Day Adventist

Protestant

Not religious

Not provided

\section{Age}

$18-25$

25-45

$45-59$

$60+$

\section{Personal qualifications}

Diploma: Clinical Officer

Assistant Medical Officer

Mmed trainee

Medical Officer

Specialist

$\mathrm{PhD}$ or other postgraduate degree

Position in the organisation

Head of Department

Principal Assistant Med Officer

Medical Officer

Senior Clinical Officer

Clinical Officer

Trainee

Not provided

Institution

A

B

C

Working experience

$<1$ year

$1-5$ years

6-10 years

$>10$ years

Continuing professional development: Last time to attend a course

This year

1-3 years ago

4-6 years ago

$7-10$ years ago

$>10$ years ago

Never

Continuing professional development: Last time to attend a workshop/event

This year

$1-3$ years ago

4-6 years ago

$7-10$ years ago

$>10$ years ago

Never

\begin{tabular}{cc}
4 & 3 \\
1 & 1 \\
1 & 1 \\
1 & 0 \\
4 & 1 \\
0 & 1 \\
5 & to attend a workshop/event \\
5 & 4 \\
2 & 2 \\
0 & 1 \\
2 & 0 \\
1 & 0 \\
1 & 0 \\
\hline
\end{tabular}

A, private not for profit; B, government regional; C, non government referral; D, private for profit.
TABLE $\mathbf{2 b}$ : The demographic distribution related to the personal identities of the laboratory workers participating in this study.

\begin{tabular}{|c|c|c|}
\hline \multirow[t]{2}{*}{ Demographic factors } & \multicolumn{2}{|c|}{ Gender } \\
\hline & Male $(N=8)$ & Female $(N=9)$ \\
\hline \multicolumn{3}{|l|}{ Ethnicity } \\
\hline Chagga & 4 & 9 \\
\hline Sukuma & 1 & 0 \\
\hline Kurya & 1 & 0 \\
\hline Haya & 1 & 0 \\
\hline Not provided & 1 & 0 \\
\hline \multicolumn{3}{|l|}{ Religion } \\
\hline Catholic & 6 & 4 \\
\hline Lutheran & 1 & 3 \\
\hline Muslim & 0 & 1 \\
\hline Seventh Day Adventist & 1 & 0 \\
\hline Not provided & 0 & 1 \\
\hline \multicolumn{3}{|l|}{ Age } \\
\hline $18-25$ & 1 & 0 \\
\hline $25-45$ & 3 & 7 \\
\hline $45-59$ & 4 & 1 \\
\hline $60+$ & 0 & 1 \\
\hline \multicolumn{3}{|l|}{ Personal qualifications } \\
\hline Laboratory attendant & 1 & 6 \\
\hline Certificate laboratory assistant & 4 & 2 \\
\hline Diploma laboratory technician & 1 & 1 \\
\hline Laboratory technologist & 0 & 0 \\
\hline Laboratory scientist & 2 & 0 \\
\hline MSc in Microbiology & 0 & 0 \\
\hline PhD or other postgraduate degree & 0 & 0 \\
\hline \multicolumn{3}{|l|}{ Position in the organisation } \\
\hline Laboratory director & 2 & 4 \\
\hline Senior employee laboratory & 3 & 3 \\
\hline Junior employee laboratory & 1 & 2 \\
\hline Trainee & 1 & 0 \\
\hline Not provided & 1 & 0 \\
\hline \multicolumn{3}{|l|}{ Institution } \\
\hline A & 1 & 2 \\
\hline B & 2 & 5 \\
\hline C & 4 & 1 \\
\hline D & 1 & 1 \\
\hline \multicolumn{3}{|l|}{ Working experience } \\
\hline$<1$ year & 2 & 0 \\
\hline $1-5$ years & 2 & 2 \\
\hline $6-10$ years & 1 & 1 \\
\hline$>10$ years & 3 & 6 \\
\hline
\end{tabular}

Continuing professional development: Last time to attend a course

This year $\quad 3 \quad 0$

$1-3$ years ago $\quad 2 \quad 2$

4-6 years ago 1

$7-10$ years ago 110

$>10$ years ago $\quad 1 \quad 5$

Not provided

1

Continuing professional development: Last time to attend a workshop and/or event This year

1-3 years ago

4-6 years ago

$7-10$ years ago

$>10$ years ago

Not provided

\begin{tabular}{ll}
4 & 3 \\
3 & 1 \\
1 & 1 \\
0 & 0 \\
0 & 2 \\
0 & 2 \\
\hline
\end{tabular}

A, private not for profit; B, government regional; C, non government referral; D, private for profit. 
treatment straight away as the waiting time to be tested in the laboratory can be very long. Clinicians sometimes agree to this request in order to save time. Laboratory staff also mentioned that nurses and sometimes patients play a role in the contact between the clinicians and laboratory staff. In some health facilities, nurses collect the sample request from the wards and are responsible for transfer of results from the laboratory back to the clinician. This supports the findings of the quantitative data analysis.

Identity: Ten (55.5\%) of the 18 clinicians and nine (52.9\%) of the 17 laboratory workers involved in the survey worked in a referral hospital. Others worked in a faith-based, public or private, for-profit facility or in a general laboratory or health facility.

\section{Personal factors}

Within the personal factors, individual and professional subfactors (qualitative data: quotes) were supportive of and in agreement with the quantitative data.

Individual factors: The personal factors of the clinicians (Table 2a) and laboratory staff (Table 2b) investigated in this study were ethnicity, religion, age and professional qualifications. Data are displayed by gender, position in the organisation and the last time a course or workshop was attended.

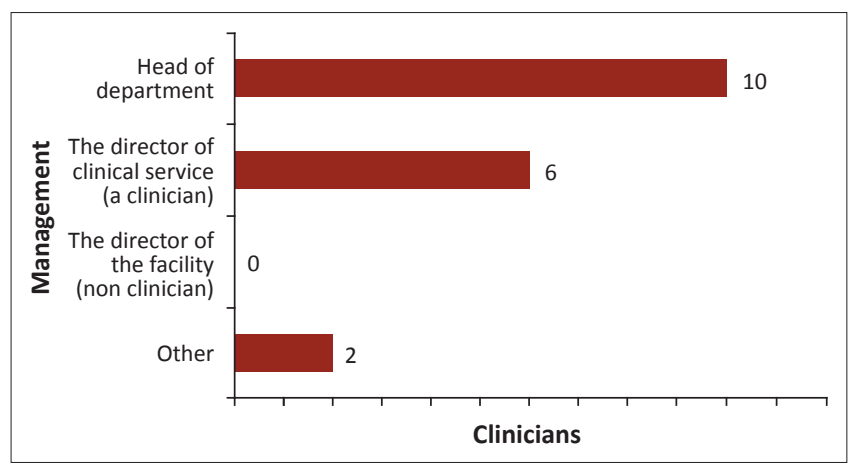

To whom does the clinician report? $(n=18)$.

Note: The two persons that mentioned 'other' indicated that these persons are medical officers.

FIGURE 1: Factors related to the management of the organisation, response of clinicians.

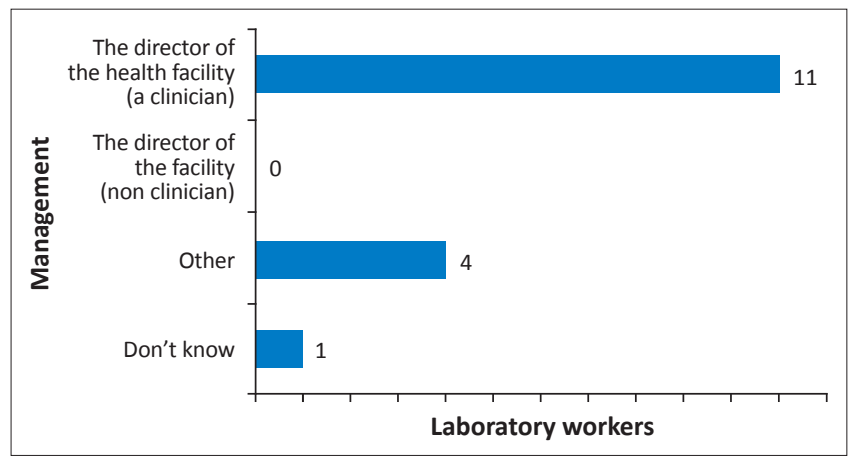

To whom does the head of the laboratory report? $(n=16)$.

Note: The four persons that indicated 'other' did not specify this. One person did not respond to this question.

FIGURE 2: Factors related to the management of the organisation, response of laboratory staff.
Professional factors: Laboratory staff members indicated that clinicians regularly devalue their services. Whilst seven of the 17 laboratory staff members $(41.1 \%)$ believed that clinicians understand what laboratory workers do and six (35.3\%) believed that clinicians know how to interpret the results when making clinical decisions, three (17.6\%) noted that clinicians do not wait for laboratory test results before starting treatment and one (5.8\%) noted that test ordering is not always specific. Furthermore, in the FGDs, nearly all of the laboratory staff members from public and faith-based organisations expressed that they lack recognition from clinicians, a sentiment also expressed by staff from the private health facility as being a contributing factor for their lack of motivation.

Further playing a part in the complicated relationship between clinicians and laboratory staff is the perceived frequency of use of test results for clinical decision making. Only six clinicians $(33.3 \%)$ and three laboratory workers $(17.6 \%)$ claimed that test results are often used in clinical decision making. One laboratory staff member $(5.8 \%)$ believed that clinicians never use the test results (Figure 3 and Figure 4).

Six of the 18 clinicians $(33.3 \%)$ do not always trust the laboratory results; $10(55.5 \%)$ mentioned that the waiting time for test results is too long; and eight $(44.4 \%)$ were not satisfied with the type of tests that can be performed and also believed that the reporting of test results is not done properly. Four clinicians (22.2\%) believed that the quality of laboratory services was weak or substandard. Several times during the FGDs, laboratory workers noted that lack of equipment contributes to poor quality output. Laboratory staff in the private hospitals pointed out the issue of lack of reagents and mentioned that expired reagents may be in use, further compromising clinicians' confidence in the laboratory test results.

In spite of the complicated relationship between clinicians and laboratory workers, a majority in both groups must interact on a daily basis (Figure 5 and Figure 6). Yet, when grievances arise, the two groups use different avenues to address them. When laboratory staff members have problems with clinicians, they often discuss them within their own professional group; however, when a clinician has a complaint, he or she will often approach the individual laboratory worker or laboratory manager. Whilst issues like these may be discussed broadly in staff meetings at most hospitals, the majority of those surveyed at the private not-for-profit hospital pointed out that the department was so small that organising meetings to discuss problems seemed unnecessary.

Poor reporting was identified as being a factor that contributed to inadequate communication between clinicians and laboratory staff. In some cases, either the handwriting was misinterpreted, or test requests or test results were incomplete. In addition, it was noted that communication only occurs between the groups when the need arises. Whilst most staff members noted that communication and positive interactions between laboratory workers and clinicians are crucial, there is no managerial support, formalised system or 
motivation to maintain regular meetings or contact between clinicians and laboratory staff.

\section{Discussion}

The main objectives of this study were to test the analytical framework; to gain a better understanding of the factors that influence the interface between clinicians and laboratory health workers; and to investigate the impact of the use of laboratory test results on the way clinicians and laboratory workers interact to deliver effective and improved healthcare. Our research results show that the roles of laboratory workers within the organisation are not determined by education levels, but by availability. According to clinicians, differences in levels of education lead to a lack of trust between clinicians and laboratory staff, impacting negatively on their collaboration and communication and creating a climate of distrust.

Poor communication between clinicians and laboratory staff further causes hostility when clinicians request a large number of tests, unaware of the high workload of the frequently understaffed laboratory. In all three phases of communication where clinicians and laboratory workers interact (pre-analytical phase: ordering of tests, sample collection; analytical phase: sample processing and analysis;

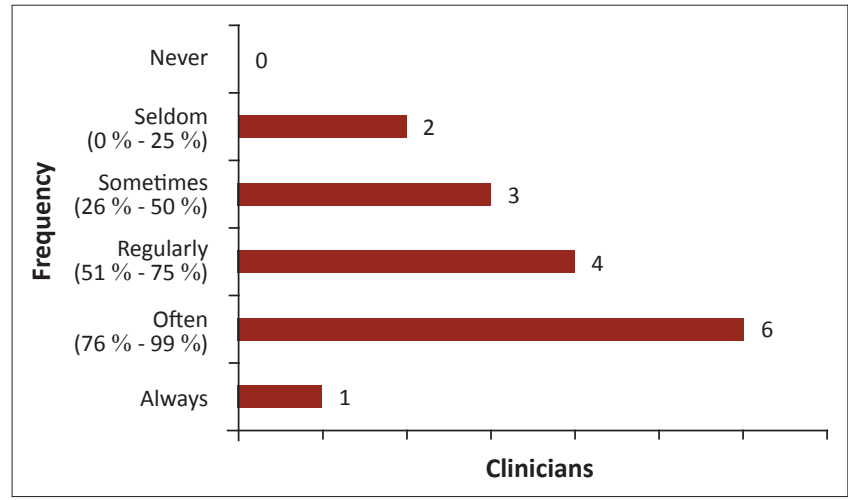

The frequency with which clinicians use the test results for clinical decision making $(n=16)$. Note: Two clinicians did not respond to this question.

FIGURE 3: Professional factors. The perceptions of clinicians regarding the frequency with which test results are used for making clinical decisions.

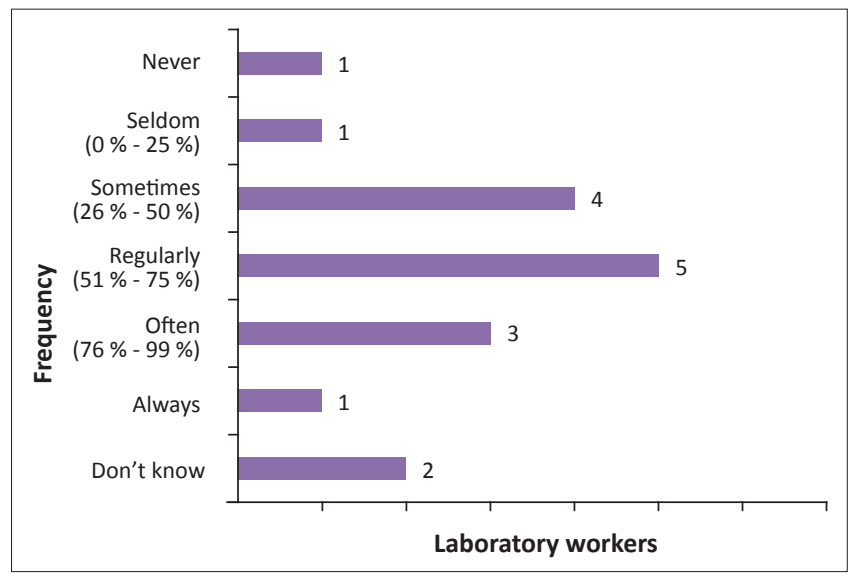

The frequency with which clinicians use the test results for clinical decision making $(n=17)$. FIGURE 4: Professional factors. The perceptions of laboratory workers regarding the frequency with which test results are used for making clinical decisions. post-analytical phase: results transfer), clinicians discuss their complaints and grievances with the laboratory staff more often than vice versa, suggesting that hierarchy plays a role in the dynamic between the two groups. Despite their different perceptions of a variety of issues, the groups agreed that clinicians were sometimes reluctant to use test results for clinical decision making. All in all, the issue of ineffective communication between clinicians and laboratory staff on patient care and worker dissatisfaction remains largely unresolved, providing a major source of frustration for staff and resulting in inefficiency in expected outputs. $5,6,7$ This study has increased the understanding of the interface between clinicians and laboratory workers and highlighted its importance in improving the quality of patient care. Scaling up data collection in a larger group of health facilities is essential with regard to quantifying our findings. This will enable hospital managers to make suggestions for improvements, such as refresher training courses that cover communication skills, as well as involving clinical and laboratory staff, nurses and patients. The research may also motivate clinicians and laboratory managers to pay more attention to the InternationalOrganization for Standardization $(\mathrm{ISO})^{18}$ stipulations regarding communication. It is hoped that the findings from this study and similar future studies

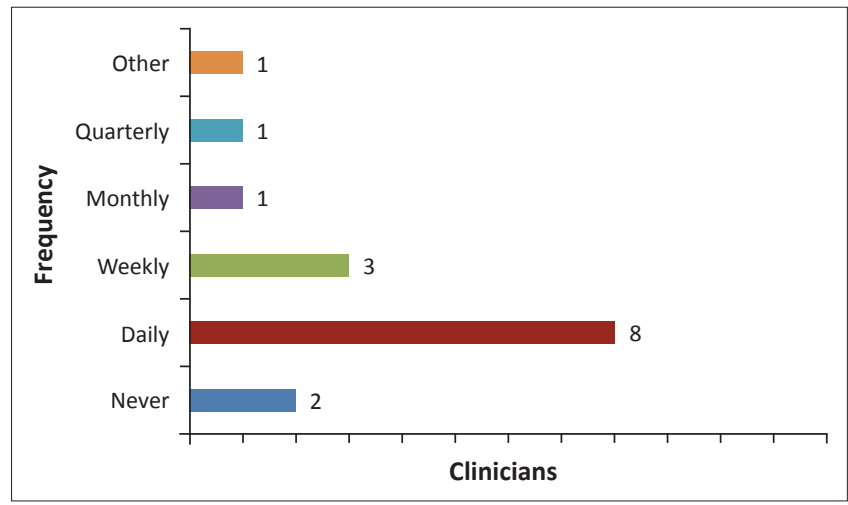

Frequency of professional contact with the laboratory workers whose services are used $(n=16)$. Note: Two clinicians did not respond to this question.

FIGURE 5: The frequency of professional contact between clinicians and laboratory workers. Eight of 16 clinicians reported having daily interactions with laboratory workers.

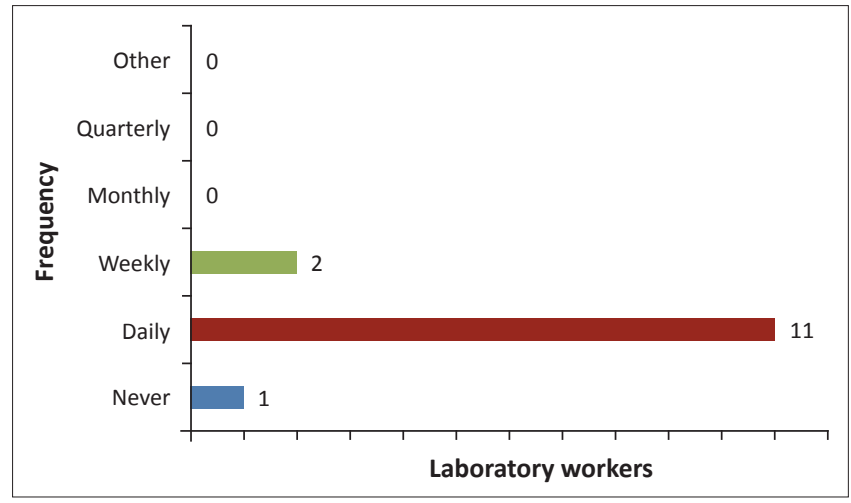

Frequency of professional contact with Clinicians that use laboratory services $(n=14)$. Note: Three laboratory workers did not respond to this question.

FIGURE 6: The frequency of professional contact between clinicians and laboratory workers. Eleven of 14 laboratory workers reported having daily professional contact with clinicians. 
will, ultimately, improve the quality of patient care and communication between clinical and laboratory staff. ${ }^{19}$

\section{Limitations of this study}

Clinicians were sometimes rushed during the interview, as patients were waiting for assistance. Only those staff who were on duty participated at the time of this study, which may have biased our results toward the perspectives of laboratory staff with lower education levels, since many more highlyeducated staff were out in the field, in training, on holiday or had resigned. The perspectives of nurses and patients were not included in this study. Laboratory staff often had difficulties in filling in the English SAQ. Some questions were not clear and allowed multiple answers, which were adjusted for in the analysis. There were inconsistencies between the questionnaires for clinicians and those for laboratory workers. The outcome of our study was based mainly on qualitative data; the quantitative components were limited. In addition, the sample size of this study was too small to draw firm conclusions. As this was a pilot study, mainly descriptive statistical methods were used. This may limit the interpretation of the data presented, but it does provide valuable information on the interface between clinicians and laboratory workers and on the effectiveness of the framework to assess this interface. These insights may be used for future studies.

\section{Conclusion}

By combining quantitative and qualitative information, some insight emerged regarding the relationship between clinicians and laboratory workers and the perspectives that contribute to their sometimes problematic interactions. This explorative study has given us additional information on factors that influence the interface between clinicians and laboratory workers and shown the effectiveness of the analytical framework. The findings and discussions also provided information for the improvement of our analytical framework, indicating the need for inclusion of nurses and patients in future studies. The findings from this study underscore the relevance of the subject: the daily struggle of hospital managers, clinicians and laboratory workers to recognise the critical role each plays in providing efficient and reliable healthcare. Performing this field study has provided information on the complexities of the interface between clinicians and laboratory staff and its impact on clinician decision making. The results justify expanding this study to a larger geographical area to include more health institutions.

\section{Acknowledgements}

We would like to thank all the staff of the hospital and clinic sites for their time and cooperation. A special thanks goes to the translators and data clerks at Kilimanjaro Clinical Research Institute (KCRI). We would also like to acknowledge Marjolein Dieleman of the Royal Tropical Institute (KIT) Health for her valuable input and critical assessment of this article. Kind thanks to Linda Oskam (KIT Biomedical Research) for her preliminary reading of the manuscript. This study would not have been possible without core funding from KCRI and KIT.

\section{Competing interests}

The authors declare that they have no financial or personal relationship(s) which may have inappropriately influenced them in writing this article.

\section{Authors' contributions}

A.vdB. (Royal Tropical Institute [KIT]), C.J.T. (Royal Tropical Institute [KIT]), Biomedical Research), D.L.M. (Kilimanjaro Christian Medical University College), E.M. (Kilimanjaro Clinical Research Institute) and M.S-dB. (Kilimanjaro Clinical Research Institute) set up the study design and developed the tools. A.vdB., C.J.T., D.L.M., E.M., J.C. (Kilimanjaro Christian Medical University College; Kilimanjaro Clinical Research Institute) and M.S-dB. performed the fieldwork. D.L.M., E.M. and M.S-dB. carried out the qualitative data analysis, A.vdB. and C.J.T. analysed the quantitative data, C.J.T. wrote the report and all authors contributed to and approved the final manuscript.

\section{References}

1. Cohen GM. Access to diagnostics in support of HIV/AIDS and tuberculosis treatment in developing countries. AIDS. 2007-21(Suppl 4):S81-S87. http://dx.doi.org/10.1097/01. aids.0000279710.47298.5c

2. Ishengoma DR, Rwegoshora RT, Mdira KY, et al. Health laboratories in the Tanga region of Tanzania: the quality of diagnostic services for malaria and other communicable diseases. Ann Trop Med Parasitol. 2009;103(5):441-453. http://dx.doi. org/10.1179/136485909X451726

3. Nkengasong JN. A shifting paradigm in strengthening laboratory health systems for . 2010;134(3):359-360. http://dx.doi.org/10.1309/AJCPY5ASUEJYQ5RK

4. Polage $C R$, Bedu-Addo G, Owusu-Ofori A, et al. Laboratory use in Ghana: physician perception and practice. Am J Trop Med Hyg. 2006;75(3):526-531.

5. Petti CA, Polage CR, Quinn TC, et al. Laboratory medicine in Africa: a barrier to effective health care. Clin Infect Dis. 2006;42(3):377-382. http://dx.doi.org/10.1086/499363

6. Chilundo B, Sundby J, Aanestad M. Analysing the quality of routine malaria data in Mozambique. Malaria Journal. 2004;3:3. http://dx.doi.org/10.1186/1475-2875-3-3

7. Leshabari MT, Muhondwa EP, Mwangu MA, et al. Motivation of health care workers in Tanzania: a case study of Muhumbili National Hospital. East Afr J Public Health. 2008;5(1):32-37. http://dx.doi.org/10.4314/eajph.v5i1.38974

8. Manongi RN, Marchant TC, Bygbjerg IC. Improving motivation among primary health care workers in Tanzania: a health worker perspective. Hum Resour Health. 2006;4:6. care workers in Tanzania: a health worker
$\mathrm{http}: / / \mathrm{dx}$.doi.org/10.1186/1478-4491-4-6

9. Garcia P, Hughes J, Carcamo C, et al. Training pharmacy workers in recognition management, and prevention of STDs: district-randomized controlled trial. Bull World Health Organ. 2003;81(11):806-814.

10. Carter J, Müller-Stöver I, Östensen H, et al. Good clinical diagnostic practice: a guide for clinicians in developing countries to the clinical diagnosis of disease and to making proper use of clinical diagnostic services. World Health Organization Regional Office or the Eastern Mediterranean: Cairo; 2005. ISBN: 978-92-9021-393-2. Available from applications.emro.who.int/dsaf/dsa236.pd

11. World Health Organization. Strategic approach for the strengthening of laboratory services for tuberculosis control, 2006-2009 [document on the Internet]. 2006 [cited 2014 Mar 30]. Available from: http://apps.who.int/iris/bitstream/10665/69303/1/ WHO_HTM_TB_2006.364_eng.pdf?ua=1

12. Butao D, Chafulumira F, Felling B, et al. Malawi: laboratory services and supply chain assessment. USAID/DELIVER PROJECT: Arlington, VA; 2009

13. Mepham SO, Squire SB, Chisuwo L, et al. Utilisation of laboratory services by health workers in a district hospital in Malawi. J Clin Pathol. 2009;62(10):935-938. http:// dx.doi.org/10.1136/jcp.2009.069062

14. May TA, Clancy M, Critchfield J, et al. Reducing unnecessary inpatient laboratory testing in a teaching hospital. Am J Clin Pathol. 2006;126(2):200-206. http://dx.do org/10.1309/WP59YM73L6CEGX2F

15. Plebani M. Exploring the iceberg of errors in laboratory medicine. Clin Chim Acta. 2009;404(1):16-23. http://dx.doi.org/10.1016/j.cca.2009.03.022

16. Van den BroekA, Tuijn CJ, Van't Klooster L, et al. Understanding the interface between clinical and laboratory staff. Afr J Lab Med. 2014;3(1), Art. In Press.

17. Varkevisser CM, Pathmanathan I, Brownlee A. Designing and conducting health systems research projects. Volume 1: Proposal development and fieldwork. KIT Publishers: Amsterdam; International Development Research Centre (IDRC); WHO Regional Office for Africa. ISBN $906832148 \mathrm{X}$.

18. International Organization for Standardization. ISO15189:2007. Medical laboratories - particular requirements for quality and competence [page on the Internet]. 2007 [cited $2014 \mathrm{Mar}$ 30]. Available from: http://www.iso.org/iso/catalogue detail?csnumber $=42641$

19. Carter JY, Lema OE, Wangai MW, et al. Laboratory testing improves diagnosis and treatment outcomes in primary health care facilities. Afr J Lab Med. 2012;1(1), Art. \#8, 6 pages. http://dx.doi.org/10.4102/ajlm.v1i1.8 